\title{
Benchmarking viromics: An in silico evaluation of metagenome-enabled estimates of viral community composition and diversity
}

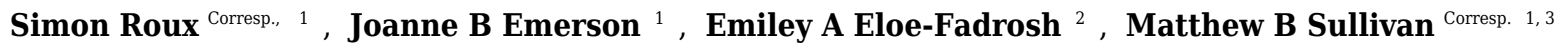 \\ 1 Department of Microbiology, Ohio State University, Columbus, Ohio, United States \\ 2 Joint Genome Institute, Department of Energy, Walnut Creek, California, United States \\ 3 Department of Civil, Environmental and Geodetic Engineering, Ohio State University, Columbus, Ohio, United States \\ Corresponding Authors: Simon Roux, Matthew B Sullivan \\ Email address: sroux@Ibl.gov, mbsulli@gmail.com
}

Background. Viral metagenomics (viromics) is increasingly used to obtain uncultivated viral genomes, evaluate community diversity, and assess ecological hypotheses. While viromic experimental methods are relatively mature and widely accepted by the research community, robust bioinformatics standards remain to be established. Here we used in silico mock viral communities to evaluate the viromic sequence-to-ecological-inference pipeline, including (i) read pre-processing and metagenome assembly, (ii) thresholds applied to estimate viral relative abundances based on read mapping to assembled contigs, and (iii) normalization methods applied to the matrix of viral relative abundances for alpha and beta diversity estimates. Results. Tools specifically designed for metagenomes, specifically metaSPAdes, MEGAHIT, and IDBA-UD, were the most effective at assembling viromes. Read pre-processing, such as partitioning, had virtually no impact on assembly output, but may be useful when hardware is limited. Viral populations with 2-5x coverage typically assembled well, whereas lesser coverage led to fragmented assembly. Strain heterogeneity within populations hampered assembly, especially when strains were closely related (average nucleotide identity, or $\mathrm{ANI} \geq 97 \%$ ) and when the most abundant strain represented $<50 \%$ of the population. Viral community composition assessments based on read recruitment were generally accurate when the following thresholds for detection were applied: $(i) \geq 10 \mathrm{~kb}$ contig lengths to define populations, (ii) coverage defined from reads mapping at $\geq 90 \%$ identity, and (ii) $\geq 75 \%$ of contig length with $\geq 1 x$ coverage. Finally, although data are limited to the most abundant viruses in a community, alpha and beta diversity patterns were robustly estimated $( \pm 10 \%)$ when comparing samples of similar sequencing depth, but more divergent (up to $80 \%$ ) when sequencing depth was uneven across the dataset. In the latter cases, the use of normalization methods specifically developed for metagenomes provided the best 
estimates. Conclusions. These simulations provide benchmarks for selecting analysis cutoffs and establish that an optimized sample-to-ecological-inference viromics pipeline is robust for making ecological inferences from natural viral communities. Continued development to better accessing RNA, rare, and/or diverse viral populations and improved reference viral genome availability will alleviate many of viromics remaining limitations. 


\title{
Benchmarking viromics: An in silico evaluation of metagenome-enabled estimates of viral community composition and diversity
}

\author{
Simon Roux ${ }^{1, \dagger, *}$, Joanne B. Emerson ${ }^{1}$, Emiley A. Eloe-Fadrosh ${ }^{2}$, Matthew B. Sullivan ${ }^{1,3, *}$ \\ ${ }^{1}$ Department of Microbiology, The Ohio State University, Columbus, OH, USA \\ ${ }^{2}$ United States Department of Energy Joint Genome Institute, Lawrence Berkeley National \\ Laboratory, Walnut Creek, CA, USA \\ ${ }^{3}$ Department of Civil, Environmental and Geodetic Engineering, The Ohio State University, \\ Columbus OH, USA \\ $\dagger$ Current address: United States Department of Energy Joint Genome Institute, Lawrence \\ Berkeley National Laboratory, Walnut Creek, CA, USA \\ * to whom correspondence should be addressed
}

\begin{abstract}
Background. Viral metagenomics (viromics) is increasingly used to obtain uncultivated viral genomes, evaluate community diversity, and assess ecological hypotheses. While viromic experimental methods are relatively mature and widely accepted by the research community, robust bioinformatics standards remain to be established. Here we used in silico mock viral communities to evaluate the viromic sequence-to-ecological-inference pipeline, including $(i)$ read pre-processing and metagenome assembly, (ii) thresholds applied to estimate viral relative abundances based on read mapping to assembled contigs, and (iii) normalization methods applied to the matrix of viral relative abundances for alpha and beta diversity estimates.
\end{abstract}

Results. Tools specifically designed for metagenomes, specifically metaSPAdes, MEGAHIT, and IDBA-UD, were the most effective at assembling viromes. Read pre-processing, such as partitioning, had virtually no impact on assembly output, but may be useful when hardware is limited. Viral populations with $2-5 x$ coverage typically assembled well, whereas lesser coverage led to fragmented assembly. Strain heterogeneity within populations hampered assembly, especially when strains were closely related (average nucleotide identity, or ANI $\geq 97 \%$ ) and when the most abundant strain represented $<50 \%$ of the population. Viral community composition assessments based on read recruitment were generally accurate when the following thresholds for detection were applied: $(i) \geq 10 \mathrm{~kb}$ contig lengths to define populations, (ii) coverage defined from reads mapping at $\geq 90 \%$ identity, and (iii) $\geq 75 \%$ of contig length with $\geq$ $1 \mathrm{x}$ coverage. Finally, although data are limited to the most abundant viruses in a community, alpha and beta diversity patterns were robustly estimated $( \pm 10 \%)$ when comparing samples of similar sequencing depth, but more divergent (up to $80 \%$ ) when sequencing depth was uneven across the dataset. In the latter cases, the use of normalization methods specifically developed for metagenomes provided the best estimates.

Conclusions. These simulations provide benchmarks for selecting analysis cut-offs and establish that an optimized sample-to-ecological-inference viromics pipeline is robust for making ecological inferences from natural viral communities. Continued development to better accessing RNA, rare, and/or diverse viral populations and improved reference viral genome availability will alleviate many of viromics remaining limitations. 


\section{Background}

Microbial communities and their associated viruses are abundant, diverse, and play key roles in Earth's ecosystems and processes (Falkowski, Fenchel \& Delong, 2008; Cobián Güemes et al., 2016). However, because most microbes and viruses remain uncultivated, and because viruses do not harbor a universal marker gene, viral ecology studies remain challenging to perform (Brum \& Sullivan, 2015; Solden, Lloyd \& Wrighton, 2016). Viral metagenomics (viromics) is a uniquely powerful tool for high-throughput analysis of uncultivated viruses (Brum \& Sullivan, 2015; Cobián Güemes et al., 2016). Initial viromics studies, despite being limited to gene-level analyses, revealed the large diversity of viral-encoded genes (Edwards \& Rohwer, 2005; Schoenfeld et al., 2008), provided first estimates of richness and functional diversity across natural viral communities (Hurwitz, Hallam \& Sullivan, 2013; Hurwitz, Brum \& Sullivan, 2015), and suggested the existence of biome-specific viral communities distributed worldwide (Rodriguez-Brito et al., 2010; Roux et al., 2012).

Thanks to recent improvements in high-throughput sequencing technologies and genome assembly, viromes now also provide the opportunity to assemble large genomes fragments (and even complete genomes) of uncultivated viruses (reviewed in Brum and Sullivan, 2015; Rose et al., 2016). Historically, in silico benchmarks of the assembly process for microbial metagenomes indicated that accurate bacterial and archaeal genomes (complete or partial) could be recovered for relatively abundant lineages given sufficient sequencing depth, but revealed potential issues including misassemblies deriving from the presence of very closely related organisms (Mavromatis et al., 2007; Mende et al., 2012; Greenwald et al., 2017; Sczyrba et al., 2017). Viral community datasets are typically processed using the same methodologies, and viral-specific benchmarks came to a similar conclusion: viral genomes can be assembled from metagenomes, but the presence of co-existing viruses with highly similar regions in their genome can lead to reduced contig size and/or chimeric contigs (Aguirre de Cárcer, Angly \& Alcamí, 2014; Vázquez-Castellanos et al., 2014; García-López, Vázquez-Castellanos \& Moya, 2015; MartinezHernandez et al., 2017; White, Wang \& Hall, 2017). However, new metagenome assembly softwares (e.g. metaSPAdes (Nurk et al., 2017)) and methods for read filtering and/or partitioning prior to assembly (e.g. khmer (Crusoe et al., 2015)) that might improve assembly quality have yet to be evaluated with viral data.

For bacteria and archaea, advances in genome binning and genome validation approaches (e.g., (Parks et al., 2015)) have significantly improved the recovery of accurately reconstructed genomes from increasingly complex environments (Wrighton et al., 2012; Sharon et al., 2013; Waldor et al., 2015; Sangwan, Xia \& Gilbert, 2016; Sczyrba et al., 2017). These methods rely on single-copy marker genes to assess genome bin completeness and "contamination" (i.e. multiple genomes in the same genome bin), two metrics critical to guide the optimization of genome binning parameters and curate the final dataset (Parks et al., 2015; Bowers et al., 2017). Unfortunately, because of the absence of universal single-copy viral marker gene, viral genome bins are much more challenging to interpret and analyze. Since viral genomes are also smaller than microbial ones and thus more frequently assembled in a single contig, viromics studies usually rely on the assembled contigs without applying any genome binning step.

For ecological analyses, a community abundance matrix of microbial OTU counts across samples is the typical starting point, and this "OTU table" is often derived from 16S rRNA gene abundances in amplicon sequencing datasets or metagenomes (Hill et al., 2003; Roesch et al., 2007; Fulthorpe et al., 2008; Fierer et al., 2011; Logares et al., 2014). Even for these relatively established microbial ecological analyses, appropriate normalization methods that account for 
92 different sequencing throughput across samples are still debated, and rarely are results compared 93 across multiple normalization methods to establish best practices (Doll et al., 2013; Paulson et 94 al., 2013; McMurdie \& Holmes, 2014). This microbial ecology pipeline also needs adjustment 95 when applied to viruses because viruses lack a universal marker gene, precluding amplicon96 based viral population abundance estimates at the community scale (although amplicon-based 97 studies have been successful for ecological analyses of specific viral lineages, e.g., (Filée et al., 2005; Goldsmith et al., 2011; Chow \& Fuhrman, 2012)). Notably, comparative genomic and ecological analysis of model systems enabled the identification of sequence-discrete populations, which represent stable ecotypes in natural viral communities (Marston \& Amrich, 2009; Gregory et al., 2016; Marston \& Martiny, 2016). Thus, in the absence of a universal viral marker gene, these genome-based populations have been proposed to be used as a viral population units (akin to a microbial operational taxonomic unit, OTU) in ecological analysis (Brum et al., 2015). Pragmatically, viral populations are derived from de novo metagenomic assemblies, with abundances estimated by metagenomic read recruitment. Ecological analyses of these contigderived abundance matrices still have to be comprehensively evaluated, although one bias specific to this approach has already been identified: counting each assembled contig as a separate OTU can lead to over-estimates of the number of different viruses in the community (Aziz et al., 2015; García-López, Vázquez-Castellanos \& Moya, 2015).

Here we used 14 in silico simulated viral metagenomes to $(i)$ compare the assembly results across different reads pre-processing methods and assemblers, both in terms of the overall genomes recovery and the number and type of errors observed, (ii) assess potential biases and identify optimal thresholds for identification and quantification of viral populations from metagenomic contigs, and (iii) determine if virome populations abundance matrices can provide reliable estimates of alpha diversity (i.e. diversity within a community) and beta diversity (i.e. differentiation between communities), even in cases where sequencing depth vary widely (up to two orders of magnitude) between samples.

\section{Methods}

\section{Mock community design}

Viral genomes were randomly selected among the complete genomes of viruses infecting bacteria or archaea in the NCBI RefSeq database (v69, 2015-02). For each mock community, the total number of viruses randomly selected (between 500 and 1,000, Table S1, Fig. S1A), as well as the parameter of the power law distribution used to model relative abundances (between 1 and 50) were varied (Fig. S1B-D). To create patterns of beta diversity across samples, the 50 most abundant viruses were homogenized within each of four sample groups, i.e., samples within a group shared 30 to 50 of their most abundant viruses, and samples between groups did not share any of their most abundant 50 viruses. This led to a clear beta diversity pattern with the mock communities clustering into four groups (Fig. S1E \& F, a PerMANOVA was performed in R with the package vegan (Oksanen et al., 2008) to verify that the sample groups were significantly different).

133

134

\section{Virome simulations}

To simulate virome sequencing for each mock community, the number of reads derived from each genome was first calculated based on the relative abundance of the genome in the mock community and the total number of reads sequenced in the virome (10 millions paired-end reads 
138 in the initial viromes, 1 million and 100,000 paired-end reads for the subsets at $10 \%$ and $1 \%$ 139 respectively). Then, NeSSM (Jia et al., 2013) was used to generate random reads (2 x 100bp) at 140 the prescribed abundances with simulated Illumina HiSeq errors.

141

142

\section{Reads processing}

Reads generated by NeSSM were first quality-controlled with Trimmomatic (Bolger, Lohse \& Usadel, 2014) with a minimum base quality threshold of 30 evaluated on sliding windows of 4 bases, and minimum read length of 50 . We opted not to evaluate different error correction softwares or to compare raw reads to quality-controlled (QC) reads, as previous studies have already provided such benchmarks for genomic assembly, which should be applicable to metagenomic assembly as well (e.g., (Yang, Chockalingam \& Aluru, 2013)).

All sets of additionally pre-processed reads were generated from these QC reads using khmer v1.4.1 (Crusoe et al., 2015), following the online protocols (http://khmerprotocols.readthedocs.io/, Fig. S2). First, a dataset of digitally normalized reads was generated, i.e. a dataset in which all reads with median k-mer abundance higher than a specified threshold were eliminated. This was done in two steps by normalizing k-mer coverage first to $20 \mathrm{x}$ then to 5x (script "normalize-by-median", dataset "Digital normalization"). The script "do-partition" was then used to partition these digitally normalized datasets, i.e. separate reads that did not connect to each other in the k-mer graphs in different bins (dataset "Partitioned reads (normalized)"). These reads partitions were then re-inflated, i.e., the original abundance of reads was restored to its value prior to digital normalization, with the script "sweep-reads" (dataset "Partitioned reads (inflated)"). Finally, three sets of reads were generated by trimming all lowabundance k-mers for highly covered reads, i.e., highly covered reads (in this case, $\geq 20 x$ ) were truncated at the first occurrence of a k-mer below a given abundance cutoff (here $\leq 2 x, \leq 5 x$, and $\leq 20 \mathrm{x}$ for the three datasets "Low k-mer filter (2x)", "Low k-mer filter (5x)", "Low k-mer filter (20x)", respectively). This was done with the script "filter-abund", with option "variablecoverage" as recommended for metagenomes.

\section{Assembly and comparison to input genomes}

The different read sets were assembled with five different assembly software tools, using metagenomic-optimized parameters (when available, Fig. S2). IDBA-UD v.1.1.1 (Peng et al., 2012) was used with the option "pre-correction" and from fasta reads (converted from fastq reads with the tool "fq2fa"). MetaSPAdes assemblies (Nurk et al., 2017) were computed from the software version 3.10.0, with the option "metagenomic" (all other options default). MEGAHIT assemblies (Li et al., 2016) were computed from version v1.0.6 with presets "meta" (all other options default). MetaVelvet assemblies (Namiki et al., 2012) were computed with software version 1.2.07 with the "discard_chimera" option selected, default parameters otherwise. Omega assemblies (Haider et al., 2014) were computed with software version 1.0.2 and minimum overlap length of 60 . Each assembler was applied to each read pool from each sample (7 read pools $\times 14$ samples $=98$ assemblies, Fig. S2), retaining all contigs $\geq 500$ bp for each assembly (Table S4).

Contigs were compared to the input genomes with nucmer (Delcher, Salzberg \& Phillippy, 2003 )(default options). When $\geq 95 \%$ of a contig's length matched an input genome at $\geq 90 \%$ nucleotide identity, that contig was considered to be a genuine assembly of the input genome. Otherwise, if a contig was similar to multiple genomes but to none over $\geq 95 \%$ of its length, it was considered a chimera. Circular contigs were detected based on identical 5' and 3' ends, as in 
184 (Roux et al., 2014). A circular contig with a length corresponding to $\geq 95 \%$ of the original 185 genome length was considered a genuine complete genome assembly, while circular contigs 186 covering less than $95 \%$ of the original genomes were considered false positives (i.e., incomplete 187 contigs incorrectly predicted as complete genome assemblies). R was used to conduct t-test when 188 comparing rate of chimeric contigs across assemblers and reads pre-processing methods, using 189 the assembly of QC reads with MEGAHIT as the control (the set of contigs with the lower 190 number of chimeras).

191

\section{Generation of the non-redundant pool of population contigs and coverage estimation}

Based on the previous benchmarks, the assemblies obtained with metaSPAdes from the QC reads were considered to be the most optimal assemblies and were used in all subsequent benchmarking analyses. Contigs from all samples were clustered with nucmer (Delcher, Salzberg $\&$ Phillippy, 2003) at $\geq 95 \%$ ANI across $\geq 80 \%$ of their lengths, as in (Brum et al., 2015; Gregory et al., 2016), to generate a pool of non-redundant "population contigs". QC reads from each sample were then mapped to these population contigs with bbmap (http://bit.ly/bbMap), with ambiguous mapping assigned to contigs at random (option ambiguous=random). A custom python script was then used to estimate the number of reads and coverage of each contig.

\section{Alpha and beta diversity estimates}

The abundance of each population contig in a given sample was estimated based on the number of reads mapping to that contig, normalized by the contig length (to account for differences in contig / genome size). Beyond the raw read counts (normalized by contig length), five abundance matrices were generated with different library size normalization methods as follow (summarized in Fig. S2):

- "Normalized": counts were divided by the total library size, i.e., the total number of QC reads in the sample, as used for example in (Brum et al., 2015). This approach is also known as "total-sum scaling".

209

210

211

212

213

214

215

216

217

218

219

220

221

222

223

224

225

226

227

228

229

- "MGSeq": counts were normalized through cumulative-sum scaling with the metagenomeSeq R package (Paulson et al., 2013). This method was specifically designed for metagenomes in which communities are under-sampled (as is the case in most viral metagenome studies), and will divide counts by a cumulative sum of count to a given percentile (as opposed to dividing by total counts as in "Normalized"). This will minimize the effects of the few highly abundant viruses potentially dominating the community, and introducing biases in relative abundances (Paulson et al., 2013).

- "EdgeR": counts were normalized using scaling factors for libraries designed to minimize the log-fold change between samples for most of the populations, computed with the edgeR R package (Robinson, McCarthy \& Smyth, 2009). This method was initially developed for count-based expression data and assumes that the relative abundances of most features (here populations) will not vary between two samples.

- "DeSeq": as with EdgeR, counts were normalized to minimize variations between samples for most populations but with a different underlying model, computed with the DESeq R package (Anders \& Huber, 2010). As with EdgeR, this method was initially developed for the detection of differentially expressed features in sequence count data analysis.

- "Rarefied": new counts were generated based on rarefied sets of reads, i.e. qualitycontrolled reads are subsampled (without replacement) to the smallest number of quality- 
230

231

232

233

234

235

236

237

238

239

240

241

242

243

244

245

246

247

248

249

250

251

252

253

254

255

256

257

258

259

260

261

262

263

264

265

266

267

268

269

270

271

272

273

274

275

controlled reads across all samples. Thus, all of the libraries are artificially set to the same size, however some data are "wasted" in the process, i.e., for the more deeply sequenced samples, some observations will not be included in the rarefied counts (McMurdie \& Holmes, 2014).

Each abundance matrix was then used to calculate alpha and beta diversity indices, namely the Shannon index, Simpson index, and pairwise Bray-Curtis dissimilarities between samples with a custom perl script. R was used to generate all plots using the ggplot2 package (Wickham, 2009), as well as the NMDS and PerMANOVA analyses, computed with the vegan package (Oksanen et al., 2008). For alpha diversity, we opted to only test indices reflecting community structure (Shannon and Simpson indexes) and not indices predicting sample richness (e.g. Chao estimators (Chao, 1984)), since the latter have been highlighted as not suitable for cases in which rare members of the community are not adequately sampled (Haegeman et al., 2013).

\section{Under-sequencing and strain heterogeneity benchmarks}

To evaluate the impact of under-sequencing on alpha and beta diversity estimates, the same pipeline (assembly with metaSPAdes from QC Reads, selection of population contigs, and estimation of alpha and beta diversity) was applied to datasets in which seven of the 14 samples were under-sequenced. Two levels of under-sequencing were tested, one in which undersequenced samples were set at $10 \%$ of the initial library size (i.e. 1,000,000 reads) and another at $1 \%$ of the initial library size $(100,000$ reads, Table S1).

To evaluate the impact of strain heterogeneity (within-population genomic diversity) on assembly success, a custom perl script was used to simulate strain variations as observed on natural populations of T4-like cyanophages (Gregory et al., 2016), i.e. a set of potentially mutated positions were determined for each new simulated strain gathering all intergenic positions, all third codons positions in protein-coding genes, and all positions in two randomly selected genes (to simulate genes undergoing diversifying selections). These simulations were based on the mock community "Sample_1", for which every genome was transformed into a population composed of a set of related strains.

For each population, three parameters selected randomly and independently:

- The total number of strains was set at 10,50 , or 100 strains simulated

- The strain divergence, controlled by a "mutation rate", i.e. the ratios of positions mutated within the set of positions identified as "potentially mutated" (see above). The other positions in the genome, not selected as potentially mutated, were mutated at a rate 100 times lower. This "mutation rate" was set at $5 \%, 10 \%$, or $20 \%$. This led to ANI between the generated strains and the original reference genomes of $97-100 \%, 95-97 \%$, and 90 $95 \%$, respectively.

- The relative abundance of individual strains within the population, sampled from a power-law distribution. The shape of the distribution was controlled by the power-law parameter, set at $0.1,1,10,100$, or 1000 . This led to the dominant (i.e. most abundant) strain representing from $1 \%$ to $100 \%$ of the population.

For each population, reads were then simulated with NeSSM (Jia et al., 2013), with the total reads generated for each population calculated based on the input coverage (as for previous simulations), and the number of reads generated from each strain calculated from the strains relative abundance. Reads were then processed as previously, i.e. quality-controlled, partitioned, or filtered, and assembled with the 5 assemblers tested using the same options as for the simulated viromes. Finally, the size of the largest contig recovered for each population was 
276 compared to the size of the largest contig recovered for the same genome without strain

277 heterogeneity, to evaluate the impact of strain heterogeneity independently from differences in

278

279

280

281

282

283

284

285

286

287

288

289

290

291

292

293

294

295

296

297

298

299

300

301

302

303

304

305

306

307

308

309

310

311

312

313

314

315

316

317

318

319

320

321

assembly efficiency between coverage levels, reads processing methods, and assemblers.

\section{Results \& Discussion}

Mock communities design

A set of 14 viral communities was designed to provide a gradient of alpha diversity and clear beta diversity patterns (Fig. S1, Table S1 \& S2). These communities were composed of 500 to 1,000 genomes (randomly sampled within bacteriophages and archaeal viruses available in NCBI RefSeq v69), with the relative abundance of individual genomes based on power law distributions with varying exponents. These simulations are thus designed to reflect a diverse viral community, as is usually observed in environmental samples (e.g., oceans, lakes, soils, or human gut), but would not correspond to viral communities dominated by a single type of virus, e.g., clinical samples associated with a specific host or epidemiological samples targeting a specific type of virus. Beyond differences in alpha diversity, these communities were also designed to organize into four "ecological" clusters, i.e., four groups of mock communities sharing more genomes within than between groups (Fig. S1). Thus, this simulated dataset allows us to evaluate the ability of virome-based population ecology approaches to recover absolute values of alpha diversity, as well as trends in alpha diversity and beta diversity patterns across samples.

Virome reads were simulated in silico with NeSSM (Jia et al., 2013) for each mock community $(10,000,000$ paired-end Illumina HiSeq reads, 2 x 100bp). Since the number of reads derived from each genome was based on its prescribed relative abundance in the community, $29.1 \%$ to $75.2 \%$ of the viral genomes in each mock community did not get "sequenced" at all (i.e., did not yield any reads). This was by design to mimic the lack of sampling for rare viruses by current sequencing efforts of environmental samples.

\section{Testing the capacity and accuracy of assembly tools}

Given metagenomic sequence data from these 14 mock communities, we first evaluated currently available assembly algorithms. To this end, five assemblers (IDBA-UD (Peng et al., 2012), MEGAHIT (Li et al., 2016), MetaVelvet (Namiki et al., 2012), Omega (Haider et al., 2014), and metaSPAdes (Nurk et al., 2017), all adapted to assemble metagenomic data) were compared to assess their ability to accurately assemble genomes of bacterial and archaeal viruses from viromes (Fig. S2). As expected, each of the assemblers successfully assembled highly covered genomes (10x or higher) and failed to assemble most low-coverage genomes (2x and lower, Fig. 1A, Fig. S3A). However, MetaVelvet and Omega required higher coverage to assemble viral genomes $(\sim 5-10 \mathrm{x})$, while IDBA-UD, MEGAHIT, and metaSPAdes routinely assembled genomes at $\sim 2-5 \mathrm{x}$ coverage (Fig. 1A, Fig. S3A). A similar trend was found when observing genome recovery in a single contig (i.e., the percentage of a genome assembled in a single contig, as opposed to the percentage of a genome assembled when cumulating all contigs). Again, IDBA-UD, MEGAHIT, and metaSPAdes were more efficient than MetaVelvet and Omega for assembling large genome fragments at lower read coverage $(\sim 2-20 \mathrm{x})$, and metaSPAdes was also better than IDBA-UD and MEGAHIT for assembling low-coverage genomes in a single large contig (Fig. 1B, Fig. S3B).

When comparing individual genome assemblies across the three best assemblers (metaSPAdes, IDBA-UD, and MEGAHIT), no clear differences could be observed in the genome recovery 
322 (Fig. S4, correlation coefficients between assemblers > 0.99). However, the percentage of each 323 genome recovered in a single contig was more variable among assemblers (Fig. S4, correlations 324 coefficients: 0.88-0.98). This comparison did not indicate that one assembler would be 325 systematically better than another, but rather that the best assembly for a given genome could come from any of these three assemblers.

Together these comparisons suggest that: (i) IDBA-UD, MEGAHIT, and metaSPAdes are currently the best available choices for maximizing assembly of viral contigs from short-read (100 bp) viromes (assembly accuracy discussed below), (ii) regardless of the choice of assembly tool, low coverage genomes $(<2 \mathrm{x})$ are under-assembled, and (iii) because assembly success varies across genomes and assemblers, multiple tools should be compared to optimally assemble desired target genomes from viromes. Overall, these results are consistent with microbial metagenomic benchmarks, which also indicated that assemblers designed specifically for metagenomes, especially metaSPAdes, MEGAHIT, and IDBA-UD, provided the best assemblies (Sczyrba et al., 2017; Vollmers, Wiegand \& Kaster, 2017).

\section{Impact of k-mer-based read filtering and partitioning on assembly}

Next, we evaluated how available read pre-processing approaches impacted genome assembly (using approaches from the khmer package and summarized in Table S3 and Fig. S2) (Crusoe et al., 2015). Briefly, beyond the reference dataset of quality controlled reads, the different methods tested were (i) trimming of reads based on low-abundance k-mers, i.e., reads are truncated at the first occurrence of a low-abundance k-mer likely originating from sequencing error, (ii) digital normalization, i.e., the removal of redundant sequences to normalize genome coverage at or under a specific value (here 5x), and (iii) read partitioning, i.e., separate assembly of the disconnected components of the k-mer graph.

Overall, and compared with the effect of the different assembly algorithms, the read preprocessing had a minimal impact on the assembly output (Fig. 1C \& D, Fig. S3 C \& D with metaSPAdes; the same observations were made with different assemblers in Fig. S5). The main effects observed were that $(i)$ digital normalization (treatments "Digital normalization" and "Partitioned reads (normalized)") led to sub-optimal assemblies, likely because differences in coverage above $5 \mathrm{x}$ are useful for assemblers to distinguish between related genomes, and (ii) trimming of low-abundance k-mers led to sub-optimal assemblies when the threshold used to define low abundance k-mers was close to the threshold used to define "abundant" reads to be trimmed (effect especially noticeable for the 20x filter, Fig. 1C \& D). Conversely, partitioning reads and keeping their coverage information (treatment "Partitioned reads (inflated)") or trimming low-abundance k-mers from high coverage reads (with thresholds of $2 \mathrm{x}$ and $5 \mathrm{x}$ ) had little effect on the assembly output, except on low-coverage genomes $(<5 \mathrm{x})$. These observations are consistent with the initial expectations of khmer's performance (Crusoe et al., 2015), although these simulations illustrate that digital normalization alone (i.e., without read partitioning and restoration of original read coverage) can lead to a sub-optimal metagenomic assembly.

\section{Errors and limitations of genome assembly from viromes}

Beyond the assembly of low-coverage genomes, which was found to be challenging for all assemblers tested, other errors are known to occur during the de novo assembly of viromes. 
367 First, chimeric contigs (i.e., contigs representing artificial constructs assembled from two or 368 more distinct genomes) were generated in each assembly, as previously noted (Aguirre de 369 Cárcer, Angly \& Alcamí, 2014; Vázquez-Castellanos et al., 2014; García-López, Vázquez370 Castellanos \& Moya, 2015). In our simulated data, these usually represented less than $2.5 \%$ of

371

372

373

374

375

376

377

378

379

380

381

382

383

384

385

386

387

388

389

390

391

392

393

394

395

396

397

398

399

400

401

402

403

404

405

406

407

408

409

410

411 the assembled datasets, and less than $5 \%$ of the large contigs $(\geq 10 \mathrm{~kb})$, but these numbers varied between assemblers and read curation methods (Fig. 2A\&B). This low number of chimeric contigs is in accordance with benchmarks of microbial metagenomes, and suggests that metagenome assemblers in general can correctly reconstruct microbial and/or viral genomes (Mende et al., 2012). For all assemblers, reads after digital normalization always yielded more chimeric contigs, which confirmed that the digital normalization step led to sub-optimal assemblies ( $\mathrm{p}$-value $<0.01)$. MEGAHIT systematically produced fewer chimeric contigs than IDBA-UD and metaSPAdes, especially for large $(\geq 10 \mathrm{~kb})$ contigs (Fig. 2B, p-value $<0.01$ ). Hence, although MEGAHIT did not assemble as many large genome fragments, the fragments that were assembled contained fewer chimeras.

Next, we investigated whether finished and closed viral genomes assemblies could be robustly identified as "circular" contigs, i.e., contigs with matching 5' and 3' ends, as previously suggested (Roux et al., 2014). The ratio of false-positive circular contigs, i.e. circular contigs that represented less than $95 \%$ of the original genome and thus likely arose from repeat regions within a genome, was not modified by read pre-processing but was different among assemblers (Fig. 2C). Specifically, 10 to $30 \%$ of the circular contigs generated by MEGAHIT and IDBA-UD did not correspond to a complete genome, while metaSPAdes assemblies rarely included any false positive ( 4 contigs, or $<2 \%$, for metaSPAdes assemblies of quality-controlled reads). This suggests that metaSPAdes circular contigs are more likely to correspond to complete genomes and that the "circularization" of a contig cannot be considered as proof of completeness for MEGAHIT and IDBA-UD contigs.

Finally, we evaluated the impact of population strain heterogeneity - i.e., the co-existence of closely related strains with distinct genomes from the same population - on virome assembly. In microbial communities, strain heterogeneity is known to considerably hamper the assembly of the corresponding genomes (Sharon et al., 2015; Martinez-Hernandez et al., 2017; Sczyrba et al., 2017). Population genetic studies of natural viral communities are however challenged by the paucity of cultivated systems that include multiple viral genomic representatives from a single population. Pragmatically, this means that although strain heterogeneity has been observed for specific model systems (Gregory et al., 2016; Marston \& Martiny, 2016), community-wide strain variations that would accurately reflect natural viral communities cannot be pulled from these data. Hence, we opted to generate a mock community using the same populations and relative abundances as Sample 1 above, but introduced some level of strain heterogeneity for each population by varying a combination of 3 parameters: $(i)$ the number of strains in the population, either low $(n=10)$, medium $(n=50)$, or high $(n=100)$, (ii) the diversity of these strains, presented as the average ANI of strains compared to the consensus population genome, either low (9095\%), medium (95-97\%), or high (97-100\%), and (iii) the evenness of the power-law distribution of strain frequency in the population, either low (dominant variant represents $75-100 \%$ of the population), medium (dominant variant $50-75 \%$ ), or high (dominant variant $<25 \%$ ). For each genome, reads were thus not generated from the reference genome sequence as before, but from a set of strains generated and sampled using a random combination of these 3 parameters. Then, the same pipeline of read processing and assembly was applied, and the size of the largest contig 
412 obtained for each population was compared to the size of the largest contig obtained in the 413 previous mock community assembly (i.e. without strain heterogeneity, Fig. 2D and Fig. S6).

414 An ANOVA was performed on the complete dataset (i.e. all combinations of assemblers and 415 read processing) to evaluate which component of strain heterogeneity impacted the assembly 416 process (see Methods). The three parameters (number of strains, strain diversity, and evenness of 417 strain distribution) significantly but differently impacted the assembly: population shape (i.e., 418 strain distribution) was the main explanatory variable of suboptimal assemblies (F-value 149.8, 419 p-value<1e-16), strain diversity was also a strong driver of assembly failures (F-value 70.4, p420 value $<1 \mathrm{e}-16$ ), while the number of strains in the populations had a more marginal effect (F-value 421 2.8, p-value 0.06). Overall, when compared to the assemblies generated without strain 422 heterogeneity, contigs were shorter for populations with an even strain distribution (i.e. dominant 423 strain $\leq 50 \%$ of the population) and/or when strains were more similar to the consensus genome 424 (i.e. average ANI to consensus $\geq 97 \%$ ) and to each other, with the combination of both leading to 425 the greater reduction in contig length (Fig. 2D). These results indicate that strain heterogeneity 426 within natural viral populations will likely be a key factor contributing to assembly success and 427 failure, and populations of evenly distributed closely related strains will be the most likely to fail 428 to assemble in virome studies. A similar trend was observed for microbial genomes in the 429 Critical Assessment of Metagenome Interpretation benchmarks, where the assembly of closely 430 related genomes (i.e. those with strain-level heterogeneity) was found to be challenging for all 431 assemblers tested, although the experimental design did not allow the evaluation of which level 432 433 434 435 and parameter of strain heterogeneity were most impactful (Sczyrba et al., 2017).

\section{Population identification and quantification}

In viral ecological studies, the next step after assembly often consists of identifying viral populations (i.e. contigs representing individual populations) and quantifying their relative abundances in each sample. We opted to use the contigs assembled with metaSPAdes from quality-controlled reads, as they represented the largest contigs overall across the different samples (despite $\sim 1 \%$ chimerism). We pooled contigs generated from all samples into a single non-redundant database (contigs were clustered at $\geq 95 \%$ of nucleotide identity across $\geq 80 \%$ of the contig length, in accordance with population genome analysis (Gregory et al., 2016)). Quality-controlled reads were then mapped to this database to estimate contig coverage across the 14 samples. Two types of thresholds were evaluated in this mapping step: $(i)$ minimum nucleotide identity for a given read to be considered mapped to a given contig, and (ii) minimum length of the contig covered to consider a contig as "detected" in a sample (Fig. S2). Reads not meeting the threshold were removed from abundance counts, and contigs not meeting the detection threshold in a given sample were given abundance values of zero for that sample in the resulting coverage table.

Considering all non-redundant contigs $\geq 500 \mathrm{bp}$ as different populations, we observed that increasing the two thresholds (read mapping identity percentage and length of contig covered) progressively decreased the sensitivity of the analysis (evaluated here as the percentage of genomes recovered among genomes which were covered $\geq 1 \mathrm{x}$ in the sample, Fig. 3A) and the false discovery rate (or FDR, which is the percentage of contigs recovered that were not part of the initial community, i.e. these genomes did not provide any reads to the simulated metagenome, Fig. 3B). However, because FDR decreased more precipitously than sensitivity, there is an optimal combination of thresholds for which FDR can be minimized and sensitivity 457 maximized. In these simulations, that optimal threshold was $\geq 75 \%$ on the contig length coverage 
458

459

460

461

462

463

464

465

466

467

468

469

470

471

472

473

474

475

476

477

478

479

480

481

482

483

484

485

486

487

488

489

490

491

492

493

494

495

496

497

498

499

500

501

502 associated with $\geq 90 \%$ nucleotide identity for the read mapping, which led to a $3 \%$ decrease in sensitivity (compared to the most permissive thresholds), but only 13\% FDR (compared to 49\% for the most permissive thresholds).

As noted by previous studies (Aziz et al., 2015; García-López, Vázquez-Castellanos \& Moya, 2015), considering all non-redundant contigs as distinct populations strongly over-estimated the total number of populations (on average, 2 to 3 contigs were counted for each individual genome, Fig. 3C). Thus, we re-analyzed our dataset using only non-redundant contigs $\geq 10 \mathrm{~kb}$ or circular as was proposed previously, and as required for taxonomic classification by gene content network-based analysis (Bolduc et al., 2017). Again, the optimal threshold combination was $\geq$ $75 \%$ of the contig length covered and $\geq 90 \%$ read mapping identity (Fig. 3 panels D,E, \& F). However, while sensitivity declined slightly $(\sim 15 \%)$ compared to the dataset including all contigs $\geq 500 \mathrm{bp}$, FDR improved drastically to $0.2 \%$, compared to $13 \%$ observed in the above analyses. Further, by increasing the stringency of the population definition, the number of contigs per genome that were counted as a population was 1.2 which is much closer to the correct number of 1 contig per genomne. More generally, increasing this contig size threshold quickly decreased the number of contig observed per genome, and most of the over-estimation observed earlier seemed to arise from contigs $<5 \mathrm{~kb}$ (Fig. S7).

In summary, we recommend that viral populations (as an operational taxonomic unit) be defined and analyzed in viromes using contigs that are $\geq 10 \mathrm{~kb}$ or circular, and only considered "detected" when the contig is covered over $\geq 75 \%$ of its length by read mapping at $\geq 90 \%$ nucleotide identity. However, we also anticipate that the data from these sensitivity analyses will help researchers tune these thresholds to match a given study's need for high sensitivity or low FDR. Importantly though, these suggestions are specific to viromes, since microbial metagenomic studies can rely on genome binning and universally conserved, single-copy marker genes to estimate more robustly the global number and completeness of the different genomes assembled (Sczyrba et al., 2017).

\section{Alpha and beta diversity estimation from virome-derived populations}

We next sought to evaluate how the variation in community structure of our 14 mock community metagenomes impacted diversity estimations, and did so using our recommended optimized population cut-offs for identifying populations and then estimating their abundances by read mapping. These population count matrices (counting either base pairs or reads mapped to each population contig) were used as input for alpha and beta diversity estimations and compared across the dataset. Notably, these matrices included only a fraction (10-33\%) of the original genomes in the dataset, as rare viral genomes were not "sequenced", and low-coverage genomes produced only small $(<10 \mathrm{~kb})$ contigs (Fig. 4A).

Before calculating any index, the read counts were first normalized by the contig length, since viral genome lengths can be highly variable ( 2 orders of magnitude, (Angly et al., 2009)). Then, to account for potential differences in library sizes, we compared five different methods: $(i)$ a simple normalization in which counts are divided by the library size, "Normalized" (ii) a method specifically designed to account for under-sampling of metagenomes, from the metagenomeSeq R package, "MGSeq" (iii and $i v$ ) two methods designed to minimize log-fold changes between samples for most of the populations, from the edgeR R package, "edgeR", and the DESeq R package, "DESeq", and (v) a rarefaction approach whereby all libraries get randomly downsampled without replacement to the size of the smallest library, "Rarefied" (Fig. S2). 
503

504

505

506

507

508

509

510

511

512

513

514

515

516

517

518

519

520

521

522

523

524

525

526

527

528

529

530

531

532

533

534

535

536

537

538

539

540

541

542

543

544

545

546

547

For both Shannon and Simpson alpha diversity indices, the values calculated from normalized count matrices were within $10 \%$ of the actual value calculated from the whole community (Fig. 4B \& C). Hence, the recovery of abundant members of the community seems to be enough to estimate alpha diversity values. Since both Shannon and Simpson indices are based on the relative abundance of individual members of the community, the three methods that applied a sample-wide correction factor (normalization by library size, MGSeq, EdgeR) all led to the same estimations, while rarefied count matrices and DESeq, which can (slightly) modify relative abundance of populations within communities, provided statistically indistinguishable estimates (Fig. 4 B \& C). Similarly, for beta diversity estimates, pairwise Bray-Curtis dissimilarities between samples calculated from normalized counts matrices were highly similar to the dissimilarities calculated from the whole communities for all normalization methods (within $15 \%$ of actual values, $p$-value $\leq 0.001$ for Mantel test comparing true and estimated dissimilarity matrices, Fig. 4D). Thus, as long as the count matrices were normalized to account for different contig lengths and library sizes, each of the five methods tested here provided reliable estimates of alpha and beta diversity.

\section{Impact of under-sequencing and possible corrections}

Finally, to help guide researchers in making decisions about under-sequenced samples, we evaluated how alpha and beta diversity estimates were impacted by such samples in a dataset. Specifically, we performed the same computations (assembly with metaSPAdes from qualitycontrolled reads, generation of a pool of dereplicated population contigs, mapping of qualitycontrolled reads and computation of normalized count matrices), but we did so with a dataset in which half of the samples were drastically under-sequenced either at 10\% (subset_10) or 1\% (subset_1) of the original sequencing depth, respectively (Table S1, Fig. S2).

Not surprisingly, under-sequenced samples resulted in fewer genomes detected (t-test, $p$-value $<1 \mathrm{e}-05$, Fig. 4A). Using the same five normalization methods to account for these differences in sequencing depth, we found that the diversity estimations were impacted. The subset_10 samples resulted in Shannon and Simpson estimations that were close (within 16\%) to the initial estimates, but the diversity estimates in the subset_1 samples varied as much as 30\% (Fig. 4 B \& C). Hence, although the different normalization methods tested here helped to compensate for some degree of under-sequencing, none was able to recover the correct values of alpha diversity when sequencing depth was highly variable and/or when some samples were significantly undersequenced.

Similarly, beta diversity patterns (evaluated as pairwise Bray Curtis dissimilarities) were not estimated as accurately with the under-sequenced samples than with the initial samples: dissimilarities estimated from subset_10 samples varied as much as $61 \%$ compared with the true dissimilarities (mean: 5.9\%), and the ones estimated from subset_1 samples varied as much as 77\% (mean: 4.4\%; Fig. 4 E \& F). Rarefaction and MGSeq were the two normalization methods most efficient at limiting these biases, as they led to maximum variations of $11.5 \%$ and $11.3 \%$ for subset_10, and $10.9 \%$ and $52.7 \%$ for subset_1, respectively. Moreover, even with the subset_1 samples, the results of an NMDS based on these normalized count matrices were still strongly correlated with the results of an NMDS based on true relative abundances (Fig. S8, $r^{2}>0.9$ for all normalization methods but "rarefied", for which the positions of two groups are switched leading to a lower $\mathrm{r}^{2}$ of 0.64 ). Hence, beta diversity trends can be recovered even when sequencing depth was highly variable. 
Although not formally evaluated through in silico benchmarks, it is very likely that microbial metagenomes with highly uneven sequencing depth would be subjected to similar biases, and the tools tested here would be expected to perform comparably on viral and microbial metagenomes, since the input data (i.e., coverage matrix) is essentially identical. Hence, the information and guidelines provided here can in all likelihood be considered relevant for microbial metagenomes as well.

\section{Current limitations of the sample-to-ecological-inference pipeline}

Overall, these benchmarks confirmed that virome-derived abundance matrices can be used in ecological studies, with two main caveats. First, absolute viral richness will likely be underestimated, because the assembly will only yield large contigs for abundant viral genotypes without evenly distributed and/or closely related strains. Hence, absolute values of richness and diversity should be interpreted with care, although once normalized, sample comparisons of these richness and diversity metrics are generally robust to differences in community complexity and sequencing depth. Second, because this approach relies on coverage as a proxy for relative abundance, only quantitative (or near-quantitative) datasets can be used as input (Duhaime et al., 2012). Notably, protocols to generate these quantitative viromes are currently available only for dsDNA and/or ssDNA viruses (Duhaime et al., 2012; Roux et al., 2016), and still remain to be developed for their RNA counterparts, although these RNA viruses might represent up to half of the viral particles in some environments (Steward et al., 2013). Thus, when interpreting viromics-based ecological studies, it is important to remember and clearly state that these reflect only the sub-part of viral communities with (ds)DNA genomes.

\section{Conclusions}

Our comparative analysis of 14 simulated viromes showed that the genome-assembly-toecological-inference viromics pipeline can efficiently and robustly identify abundant viruses and recover trends in alpha and beta diversity. As viromics becomes routine in viral ecology, the approaches underlined here (both the tools and thresholds used) offer an initial set of "best practices" for data analysis.

Moving forward, increased library size and number associated with improved genome recovery from metagenomes will undoubtedly lead to an unprecedented catalog of uncultivated viral genomes (e.g. 125,000 released in a single study (Paez-Espino et al., 2016)). These will be complemented by viral genomes obtained from other methods, such as single-virus sequencing, which can access less dominant viruses and those with high strain heterogeneity (MartinezHernandez et al., 2017). As standards emerge, such uncultivated viral genomes will migrate toward specifically-designed databases (e.g. IMG/VR (Paez-Espino et al., 2016)), and viral ecological studies will be greatly improved by these centralized reference genome data. Beyond improved references (which will also need to include uncultivated RNA viruses), viromics will need to advance from relative abundance estimations to absolute quantification of viral populations, likely coupled with "ground-truthing" provided by quantitative, lineage-specific molecular methods such as phageFISH, polonies, microarrays, or microfluidic PCR (Tadmor et al., 2011; Allers et al., 2013; Martínez-García et al., 2014). Once in-hand, such approaches should enable researchers to address long-standing questions in the viral ecology field, and more fully bring viruses into predictive ecological models across Earth's ecosystems. 


\section{List of abbreviations}

593 ANI: Average Nucleotide Identity

594 ANOVA: ANalysis Of Variance

595 FDR: False Discovery Rate

596 NMDS: Non-metric MultiDimensional Scaling

597 OTU: Operational Taxonomic Unit

598 QC: Quality-controlled (for reads)

599

600

\section{Declaration}

601

602

Availability of data and material

603 The datasets generated for this study are available at

604 http://datacommons.cyverse.org/browse/iplant/home/shared/iVirus/Virome pipeline benchmark

605 The scripts used in this study are available at

606 https://bitbucket.org/MAVERICLab/benchmarking_viromics

607

608 Acknowledgements

609 High performance computing resources were provided by the Ohio Supercomputer Center, and

610 the National Energy Research Scientific Computing Center supported by the Office of Science of 611 the US Department of Energy.

612

613 
614 References

615 Aguirre de Cárcer D., Angly FE., Alcamí A. 2014. Evaluation of viral genome assembly and 616 diversity estimation in deep metagenomes. BMC Genomics 2014 15:1 15:e368. DOI: 10.1186/1471-2164-15-989.

618

619

Allers E., Moraru C., Duhaime MB., Beneze E., Solonenko N., Canosa JB., Amann R., Sullivan MB. 2013. Single-cell and population level viral infection dynamics revealed by phageFISH, a method to visualize intracellular and free viruses. Environmental

623

Anders S., Huber W. 2010. Differential expression analysis for sequence count data. Genome Biology 11:R106. DOI: 10.1186/gb-2010-11-10-r106.

Angly FE., Willner D., Prieto-Davó A., Edwards RA., Schmieder R., Vega-Thurber R., Antonopoulos DA., Barott K., Cottrell MT., Desnues C., Dinsdale EA., Furlan M., Haynes M., Henn MR., Hu Y., Kirchman DL., McDole T., McPherson JD., Meyer F., Miller RM., Mundt E., Naviaux RK., Rodriguez-Mueller B., Stevens R., Wegley L., Zhang L., Zhu B.,

Aziz RK., Dwivedi B., Akhter S., Breitbart M., Edwards RA. 2015. Multidimensional metrics Rohwer F. 2009. The GAAS metagenomic tool and its estimations of viral and microbial average genome size in four major biomes. PLoS computational biology 5:e1000593. DOI:

Bolger AM., Lohse M., Usadel B. 2014. Trimmomatic: A flexible trimmer for Illumina sequence

Bowers RM., Kyrpides NC., Stepanauskas R., Harmon-Smith M., Schulz F., Doud D., Reddy TBK., Jarett J., Rivers AR., Eloe-Fadrosh EA., Tringe SG., Ivanova N., Copeland A., Clum A., Becraft ED., Malmstrom RR., Birren B., Schriml L., Podar M., Bork P., Weinstock GM., Banfield JF., Garrity GM., Hugenholtz P., Parks DH., Tyson GW., Rinke C., Dodsworth J a., Yooseph S., Sutton G., Yilmaz P., Glockner FO., Meyer F., Gilbert JA., Nelson WC., Hallam SJ., Jungbluth SP., Ettema TJG., Tighe S., Konstantinidis KT., Liu WT., Baker BJ., Rattei T., Eisen JA., Hedlund BP., Mcmahon KD., Fierer N., Knight R., Finn RD., Karsch-Mizrachi I., Eren A., Woyke T. 2017. Minimum information about a single amplified genome (MISAG) and a metagenome-assembled genome (MIMAG) of bacteria and archaea. Nature Biotechnology in press. 
649 Brum J., Ignacio-Espinoza J., Roux S., Doulcier G., Acinas SG., Alberti A., Chaffron S., Cruaud 650 C., de Vargas C., Gasol JM., Gorsky G., Gregory AC., Ogata H., Pesant S., Poulos BT., 651 Schwenck SM., Speich S., Dimier C., Kandels-Lewis S., Picheral M., Searson S., 652 Coordinators TO., Bork P., Bowler C., Sunagawa S., Wincker P., Karsenti E., Sullivan MB. 653 654 2015. Patterns and ecological drivers of ocean viral communities. Science 348:1261498-1-

Cobián Güemes AG., Youle M., Cantú VA., Felts B., Nulton J., Rohwer F. 2016. Viruses as

663

664

Delcher AL., Salzberg SL., Phillippy AM. 2003. Using MUMmer to identify similar regions in

Doll HM., Armitage DW., Daly R a., Emerson JB., Goltsman DSA., Yelton AP., Kerekes J.,

Firestone MK., Potts MD. 2013. Utilizing novel diversity estimators to quantify multiple

Chow CET., Fuhrman JA. 2012. Seasonality and monthly dynamics of marine myovirus communities. Environmental Microbiology 14:2171-2183. DOI: 10.1111/j.14622920.2012.02744.x.

Crusoe MR., Alameldin HF., Awad S., Boucher E., Caldwell A., Cartwright R., Charbonneau A., Constantinides B., Edvenson G., Fay S., Fenton J., Fenzl T., Fish J., Garcia-Gutierrez L., Garland P., Gluck J., González I., Guermond S., Guo J., Gupta A., Herr JR., Howe A., Hyer A., Härpfer A., Irber L., Kidd R., Lin D., Lippi J., Mansour T., McA’Nulty P., McDonald E., Mizzi J., Murray KD., Nahum JR., Nanlohy K., Nederbragt AJ., Ortiz-Zuazaga H., Ory J., Pell J., Pepe-Ranney C., Russ ZN., Schwarz E., Scott C., Seaman J., Sievert S., Simpson J., Skennerton CT., Spencer J., Srinivasan R., Standage D., Stapleton JA., Steinman SR., Stein J., Taylor B., Trimble W., Wiencko HL., Wright M., Wyss B., Zhang Q., Zyme E., Brown CT. 2015. The khmer software package: enabling efficient nucleotide sequence analysis. F1000Research 4:900. DOI: 10.12688/f1000research.6924.1. large sequence sets. Current protocols in bioinformatics 00:10.3:10.3.1-10.3.18. DOI: 10.1002/0471250953.bi1003s00. 10.1186/1471-2180-13-259.

Duhaime MB., Deng L., Poulos BT., Sullivan MB. 2012. Towards quantitative metagenomics of wild viruses and other ultra-low concentration DNA samples: a rigorous assessment and 
684

685

686

687

688

689

690

691

692

693

694

695

696

697

698

699

700

701

702

703

704

705

706

707

708

709

710

711

712

713

714

715

716

optimization of the linker amplification method. Environmental microbiology 14:2526-37. DOI: $10.1111 /$ j.1462-2920.2012.02791.x.

Edwards RA., Rohwer F. 2005. Viral metagenomics. Nature Reviews Microbiology 3:504-510.

Falkowski PG., Fenchel T., Delong EF. 2008. The Microbial Engines That Drive Earth's Biogeochemical Cycles. Science 320:1034-9. DOI: 10.1126/science.1153213.

Fierer N., McCain C., Meir P., Zimmerman M., Rapp JM., SIlman MR., Knight R. 2011. Microbes do not follow the elevational diversity patterns of plants and animals. Ecology 92:2013-2019.

Filée J., Tétart F., Suttle CA., Krisch HM. 2005. Marine T4-type bacteriophages, a ubiquitous component of the dark matter of the biosphere. Proceedings of the National Academy of Sciences of the United States of America 102:12471-6. DOI: 10.1073/pnas.0503404102.

Fulthorpe RR., Roesch LFW., Riva A., Triplett EW. 2008. Distantly sampled soils carry few species in common. Isme Journal 2:901-910. DOI: 10.1038/ismej.2008.55.

García-López R., Vázquez-Castellanos JF., Moya A. 2015. Fragmentation and Coverage Variation in Viral Metagenome Assemblies, and Their Effect in Diversity Calculations. Frontiers in bioengineering and biotechnology 3:141. DOI: 10.3389/fbioe.2015.00141.

Goldsmith DB., Crosti G., Dwivedi B., McDaniel LD., Varsani A., Suttle CA., Weinbauer MG., Sandaa R-AA., Breitbart M. 2011. Development of phoH as a novel signature gene for assessing marine phage diversity. Applied and environmental microbiology 77:7730-9. DOI: 10.1128/AEM.05531-11.

Greenwald WW., Klitgord N., Seguritan V., Yooseph S., Venter JC., Garner C., Nelson KE., Li W. 2017. Utilization of defined microbial communities enables effective evaluation of meta-genomic assemblies. BMC Genomics 18:296. DOI: 10.1186/s12864-017-3679-5.

Gregory AC., Solonenko SA., Ignacio-Espinoza JC., LaButti K., Copeland A., Sudek S., Maitland A., Chittick L., dos Santos F., Weitz JS., Worden AZ., Woyke T., Sullivan MB. 2016. Genomic differentiation among wild cyanophages despite widespread horizontal gene transfer. BMC Genomics 17:930. DOI: 10.1186/s12864-016-3286-x.

Haegeman B., Hamelin J., Moriarty J., Neal P., Dushoff J., Weitz JS. 2013. Robust estimation of microbial diversity in theory and in practice. The ISME journal 7:1092-101. DOI: 10.1038/ismej.2013.10.

Haider B., Ahn TH., Bushnell B., Chai J., Copeland A., Pan C. 2014. Omega: An Overlap-graph de novo Assembler for Metagenomics. Bioinformatics 30:2717-2722. DOI: 10.1093/bioinformatics/btu395. 
717 Hill TCJ., Walsh K a., Harris J a., Moffett BF. 2003. Using ecological diversity measures with 718 bacterial communities. FEMS Microbiol Ecol 43:1-11. DOI: FEM1 [pii] \n10.1111/j.15746941.2003.tb01040.x.

720

721

722

723

724

725

726

727

728

729

730

731

732

733

734

735

736

737

738

739

740

741

742

743

744

745

746

747

748

749

750

751

Hurwitz BL., Brum JR., Sullivan MB. 2015. Depth-stratified functional and taxonomic niche specialization in the "core" and "flexible" Pacific Ocean Virome. The ISME Journal 9:47284. DOI: 10.1038 /ismej.2014.143.

Hurwitz BL., Hallam SJ., Sullivan MB. 2013. Metabolic reprogramming by viruses in the sunlit and dark ocean. Genome biology 14:R123. DOI: 10.1186/gb-2013-14-11-r123.

Jia B., Xuan L., Cai K., Hu Z., Ma L., Wei C. 2013. NeSSM: a Next-generation Sequencing Simulator for Metagenomics. PloS one 8:e75448. DOI: 10.1371/journal.pone.0075448.

Li D., Luo R., Liu CM., Leung CM., Ting HF., Sadakane K., Yamashita H., Lam TW. 2016. MEGAHIT v1.0: A fast and scalable metagenome assembler driven by advanced methodologies and community practices. Methods 102:3-11. DOI: 10.1016/j.ymeth.2016.02.020.

Logares R., Sunagawa S., Salazar G., Cornejo-Castillo FM., Ferrera I., Sarmento H., Hingamp P., Ogata H., de Vargas C., Lima-Mendez G., Raes J., Poulain J., Jaillon O., Wincker P., Kandels-Lewis S., Karsenti E., Bork P., Acinas SG. 2014. Metagenomic 16S rDNA Illumina tags are a powerful alternative to amplicon sequencing to explore diversity and structure of microbial communities. Environmental Microbiology 16:2659-2671. DOI: $10.1111 / 1462-2920.12250$.

Marston MF., Amrich CG. 2009. Recombination and microdiversity in coastal marine cyanophages. Environmental microbiology 11:2893-903. DOI: 10.1111/j.14622920.2009.02037.x.

Marston MF., Martiny JBH. 2016. Genomic diversification of marine cyanophages into stable ecotypes. Environmental Microbiology 18:4240-4253. DOI: 10.1111/1462-2920.13556.

Martínez-García M., Santos F., Moreno-Paz M., Parro V., Antón J. 2014. Unveiling viral-host interactions within the "microbial dark matter." Nature Communications 5:1-8. DOI: 10.1038/ncomms5542.

Martinez-Hernandez F., Fornas O., Lluesma M., Bolduc B., Cruz M., Martinez Martinez J., Anton J., Gasol J., Rosselli R., Rodriguez-Valera R., Sullivan MB., Acinas SG., MartinezGarcia M. 2017. Single-virus genomics reveals hidden cosmopolitan and abundant viruses. Nature communications 8:15892. DOI: 10.1038/ncomms15892.

Mavromatis K., Ivanova N., Barry K., Shapiro H. 2007. Use of simulated data sets to evaluate the fidelity of metagenomic processing methods. Nature methods 4:495-500. DOI: 10.1038/NMETH1043. 
752 McMurdie PJ., Holmes S. 2014. Waste Not, Want Not: Why Rarefying Microbiome Data Is

753 Inadmissible. PLoS Computational Biology 10. DOI: 10.1371/journal.pcbi.1003531.

754 Mende DR., Waller AS., Sunagawa S., Järvelin AI., Chan MM., Arumugam M., Raes J., Bork P.

755 2012. Assessment of metagenomic assembly using simulated next generation sequencing

756 data. PLoS ONE 7. DOI: 10.1371/journal.pone.0031386.

757

758

759

760

761

762

763

764

765

766

767

768

769

770

771

772

773

774

775

776

777

778

779

780

781

782

783

784

785

Namiki T., Hachiya T., Tanaka H., Sakakibara Y. 2012. MetaVelvet: an extension of Velvet assembler to de novo metagenome assembly from short sequence reads. Nucleic acids research 40:e155. DOI: 10.1093/nar/gks678.

Nurk S., Meleshko D., Korobeynikov A., Pevzner PA. 2017. metaSPAdes: a new versatile metagenomic assembler. Genome Research. DOI: 10.1101/gr.213959.116.

Oksanen J., Kindt R., Legendre P., O’Hara B., Simpson GL., Solymos P., Stevens MHH., Wagner H. 2008. The vegan Package.

Paez-Espino D., Chen I-MA., Palaniappan K., Ratner A., Chu K., Szeto E., Pillay M., Huang J., Markowitz VM., Nielsen T., Huntemann M., K Reddy TB., Pavlopoulos GA., Sullivan MB., Campbell BJ., Chen F., McMahon K., Hallam SJ., Denef V., Cavicchioli R., Caffrey SM., Streit WR., Webster J., Handley KM., Salekdeh GH., Tsesmetzis N., Setubal JC., Pope PB., Liu W-T., Rivers AR., Ivanova NN., Kyrpides NC. 2016. IMG/VR: a database of cultured and uncultured DNA Viruses and retroviruses. Nucleic acids research 45:gkw1030. DOI: 10.1093/nar/gkw1030.

Parks DH., Imelfort M., Skennerton CT., Hugenholtz P., Tyson GW. 2015. CheckM: assessing the quality of microbial genomes recovered from. Genome Research 25:1043-55. DOI: 10.1101/gr.186072.114.

Paulson JN., Stine OC., Bravo HC., Pop M. 2013. Differential abundance analysis for microbial marker-gene surveys. Nature methods 10:1200-2. DOI: 10.1038/nmeth.2658.

Peng Y., Leung HCM., Yiu SM., Chin FYL. 2012. IDBA-UD: a de novo assembler for singlecell and metagenomic sequencing data with highly uneven depth. Bioinformatics 28:14201428 .

Robinson MD., McCarthy DJ., Smyth GK. 2009. edgeR: A Bioconductor package for differential expression analysis of digital gene expression data. Bioinformatics 26:139-140. DOI: 10.1093/bioinformatics/btp616.

Rodriguez-Brito B., Li L., Wegley L., Furlan M., Angly F., Breitbart M., Buchanan J., Desnues C., Dinsdale E., Edwards R., Felts B., Haynes M., Liu H., Lipson D., Mahaffy J., MartinCuadrado AB., Mira A., Nulton J., Pasić L., Rayhawk S., Rodriguez-mueller J., RodriguezValera F., Salamon P., Srinagesh S., Thingstad TF., Tran T., Thurber RV., Willner D., 
786

787

788

789

790

791

792

793

794

795

796

797

798

799

800

801

802

803

804

805

806

807

808

809

810

811

812

813

814

815

816

817

818

819

820

Youle M., Rohwer F. 2010. Viral and microbial community dynamics in four aquatic environments. The ISME journal 4:739-51. DOI: 10.1038/ismej.2010.1.

Roesch L., Fulthorpe R., Riva A., Casella G., Hadwin A., Kent A., Daroub S., Camargo F., Farmerie W., Triplett E. 2007. Pyrosequencing enumerates and contrasts soil microbial diversity. The ISME Journal 1:283-290. DOI: 10.1038/ismej.2007.53.Pyrosequencing.

Rose R., Constantinides B., Tapinos A., Robertson DL., Prosperi M. 2016. Challenges in the analysis of viral metagenomes. Virus Evolution 2:vew022. DOI: 10.1093/ve/vew022.

Roux S., Enault F., Robin A., Ravet V., Personnic S., Theil S., Colombet J., Sime-Ngando T., Debroas D. 2012. Assessing the Diversity and Specificity of Two Freshwater Viral Communities through Metagenomics. PLoS One 7:e33641.

Roux S., Tournayre J., Mahul A., Debroas D., Enault F. 2014. Metavir 2: new tools for viral metagenome comparison and assembled virome analysis. BMC Bioinformatics 15:1-12.

Roux S., Solonenko NE., Dang VT., Poulos BT., Schwenck SM., Goldsmith DB., Coleman ML., Breitbart M., Sullivan MB. 2016. Towards quantitative viromics for both double-stranded and single-stranded DNA viruses. PeerJ 4:e2777. DOI: 10.7717/peerj.2777.

Sangwan N., Xia F., Gilbert JA. 2016. Recovering complete and draft population genomes from metagenome datasets. Microbiome 4:8. DOI: 10.1186/s40168-016-0154-5.

Schoenfeld T., Patterson M., Richardson PM., Wommack KE., Young M., Mead D. 2008. Assembly of viral metagenomes from yellowstone hot springs. Applied and environmental microbiology 74:4164-4174.

Sczyrba A., Hofmann P., Belmann P., Koslicki D., Dröge J., Gregor I., Majda S., Fiedler J., Dahms E., Bremges A., Fritz A., Garrido-oter R., Jørgensen S., Shapiro N., Blood PD., Gurevich A., Hansen LH., Sørensen SJ., Chia BKH., Denis B., Froula JL., Wang Z., Egan R., Kang DD., Singer W., Jain C., Strous M., Klingenberg H., Meinicke P., Barton M., Lingner T., Lin H., Liao Y., Silva GZ., Cuevas DA., Edwards RA., Saha S., Vitor C., Renard BY., Hill CM., Pop M., Goeker M., Kyrpides N., Woyke T., Vorholt J., Rubin EM., Darling AE., Rattei T., Alice C. 2017. Critical Assessment of Metagenome Interpretation a comprehensive benchmark of computational metagenomics software. bioRxiv. DOI: $10.1101 / 099127$.

Sharon I., Morowitz MJ., Thomas BC., Costello EK., Relman DA., Banfield JF. 2013. Time series community genomics analysis reveals rapid shifts in bacterial species, strains, and phage during infant gut colonization. Genome Research 23:111-120. DOI: 10.1101/gr.142315.112.

Sharon I., Kertesz M., Hug LA., Pushkarev D., Blauwkamp TA., Castelle CJ., Amirebrahimi M., Thomas BC., Burstein D., Tringe SG., Williams KH., Banfield JF. 2015. Accurate, multi-kb 
821

822

823

824

825

826

827

828

829

830

831

832

833

834

835

836

837

838

839

840

841

842

843

844

845

846

847

848

849

850

851

852

853

854

855

reads resolve complex populations and detect rare microorganisms. Genome Research 25:534-543. DOI: 10.1101/gr.183012.114.

Solden L., Lloyd K., Wrighton K. 2016. The bright side of microbial dark matter: Lessons learned from the uncultivated majority. Current Opinion in Microbiology 31:217-226. DOI: 10.1016/j.mib.2016.04.020.

Steward GF., Culley AI., Mueller JA., Wood-Charlson EM., Belcaid M., Poisson G. 2013. Are we missing half of the viruses in the ocean? The ISME journal 7:672-679. DOI: 10.1038/ismej.2012.121.

Tadmor AD., Ottesen EA., Leadbetter JR., Phillips R., Gallant JA., Cramer P., Weintraub H., Rich A., Maas S., Platas AA., Hawley DK., Yuzenkova Y., Severinov K., Yano T., Kawabata H., Ueda H., Suzuki T., Scott J., Anant S., Navaratnam N., Nicklen S., Coulson AR., Hamilton CE., Mwangi MM., Dewell S., Papavasiliou FN., Gish W., Miller W., Myers EW., Lipman DJ., Cox J., Mann M., Patt S., Schrey M., Rich A., Williams MA., Mulligan RM. 2011. Probing Individual Environmental Bacteria for Viruses by Using Microfluidic Digital PCR. Science 333:58-62. DOI: 10.1126/science.1200758.

Vázquez-Castellanos JF., García-López R., Pérez-Brocal V., Pignatelli M., Moya A. 2014. Comparison of different assembly and annotation tools on analysis of simulated viral metagenomic communities in the gut. BMC genomics 15:37. DOI: 10.1186/1471-2164-1537.

Vollmers J., Wiegand S., Kaster AK. 2017. Comparing and evaluating metagenome assembly tools from a microbiologist's perspective - Not only size matters! PLoS ONE 12:1-31. DOI: 10.1371/journal.pone.0169662.

Waldor MK., Tyson G., Borenstein E., Ochman H., Moeller A., Finlay BB., Kong HH., Gordon JI., Nelson KE., Dabbagh K., Smith H. 2015. Where Next for Microbiome Research? PLoS Biology 13:1-9. DOI: 10.1371/journal.pbio.1002050.

White DJ., Wang J., Hall RJ. 2017. Assessing the Impact of Assemblers on Virus Detection in a De Novo Metagenomic Analysis Pipeline. Journal of Computational Biology 24:cmb.2017.0008. DOI: $10.1089 / \mathrm{cmb} .2017 .0008$.

Wickham H. 2009. ggplot2: Elegant Graphics for Data Analysis. Springer Publishing Company.

Wrighton KC., Thomas BC., Sharon I., Miller CS., Castelle CJ., Verberkmoes NC., Wilkins MJ., Hettich RL., Lipton MS., Williams KH., Long PE., Banfield JF. 2012. Fermentation, Hydrogen, and Sulfur Metabolism in Multiple Uncultivated Bacterial Phyla. Science 337:1661-1666.

Yang X., Chockalingam SP., Aluru S. 2013. A survey of error-correction methods for nextgeneration sequencing. Briefings in Bioinformatics 14:56-66. DOI: 10.1093/bib/bbs015. 


\section{Figure 1}

Figure 1. Influence of assembly software and read curation on genome recovery.

All plots display the input coverage on the $\mathrm{x}$-axis, and either the cumulated genome recovery across all contigs $(A \& C)$ or the highest genome recovery by a single contig $(B \& D)$ on the $y$ axis. Panels A \& B display a comparison of assemblers applied to quality-controlled (QC) reads. Panels $C \& D$ present a comparison of read pre-processing methods, all assembled with metaSPAdes. Comparable plots for reads assembled with the other assemblers are available in Fig. S5.

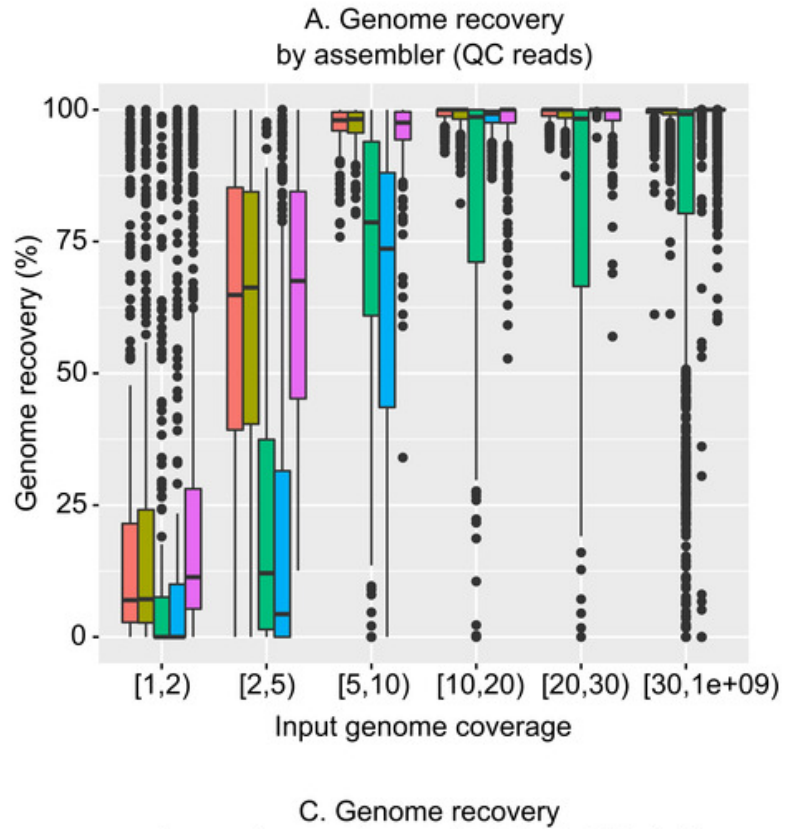

by read processing method (metaSPAdes)

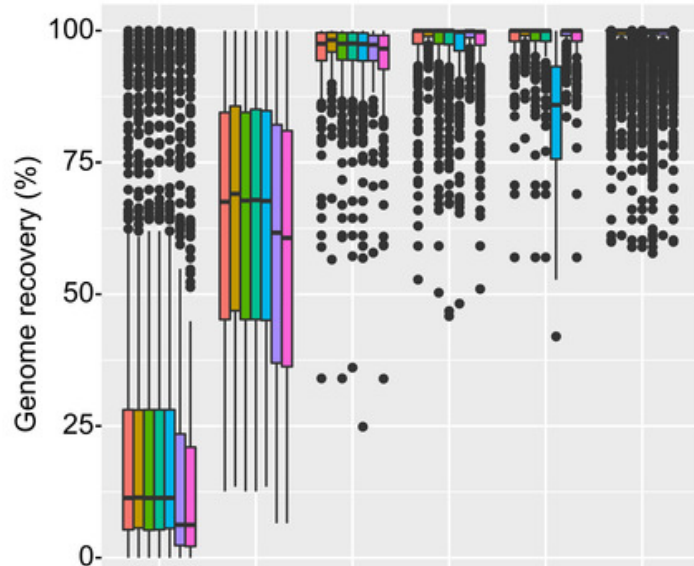

$[1,2) \quad[2,5) \quad[5,10)[10,20)[20,30)[30,1 e+09)$ PeerJ reviewingutbeheh6201 zo06rtge78:1:0:NEW 18 Aug 2017)
B. Genome recovery in a single contig by assembler ( $Q C$ reads)

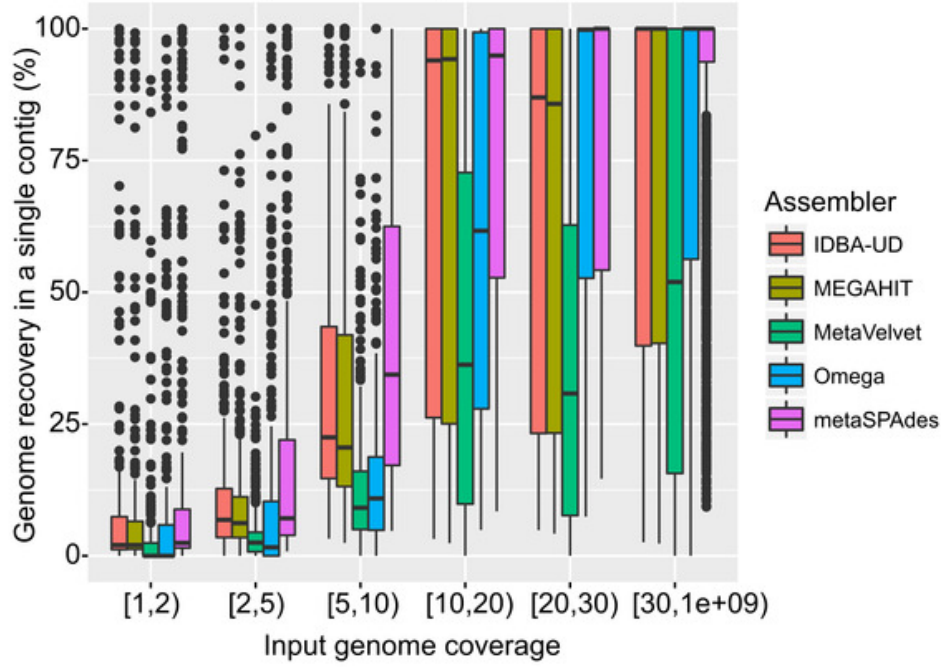

D. Genome recovery in a single contig by read processing method (metaSPAdes)

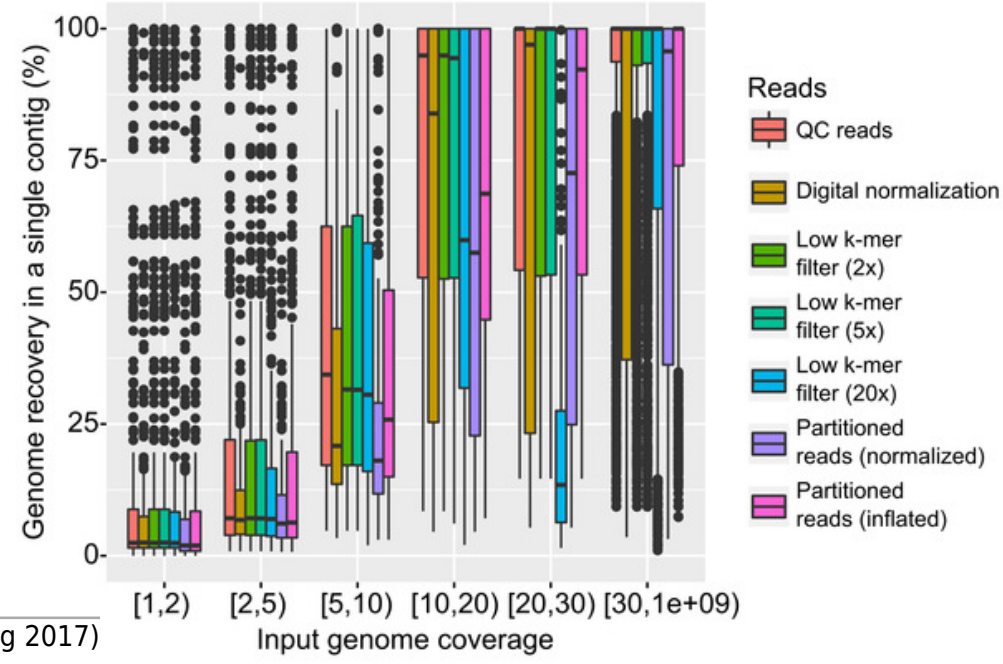




\section{Figure 2}

Figure 2. Types and frequency of errors observed in genome assembly from viral metagenomes.

A. Percentage of chimeric contigs (i.e. contigs originating from two distinct genomes) across all assembled sequences, by assembler (x-axis) and read curation method (colors). B. Percentage of chimeric contigs among large $(\geq 10 \mathrm{~kb})$ contigs, by assembler ( $\mathrm{x}$-axis) and read curation method (colors). C. Percentage of false-positive circular contigs, i.e. contigs identified as circular (matching 5' and $3^{\prime}$ ends) but representing $95 \%$ or less of the original genome, by assembler ( $\mathrm{x}$-axis) and read curation method (color). D. Impact of strain heterogeneity (i.e. presence of multiple strains from the same population) on the assembly efficiency. These tests were computed on one mock community (Sample_1), for which each reference genome was replaced with a set of related strains with varying divergence and relative abundances. The $y$-axis represents the ratio between the largest contig assembled for a genome when strain heterogeneity is introduced and the same parameter without strain heterogeneity (i.e. previous assemblies of the same Sample_1). Populations are grouped based on the two main parameters explaining assembly inefficiency: proportion of the most abundant strain in the population (bottom) and divergence of strains in the population (top). Data presented here include assemblies from QC reads with IDBA-UD, MEGAHIT, and metaSPAdes, while the full set of parameters and approaches tested are presented in Fig. S6. 

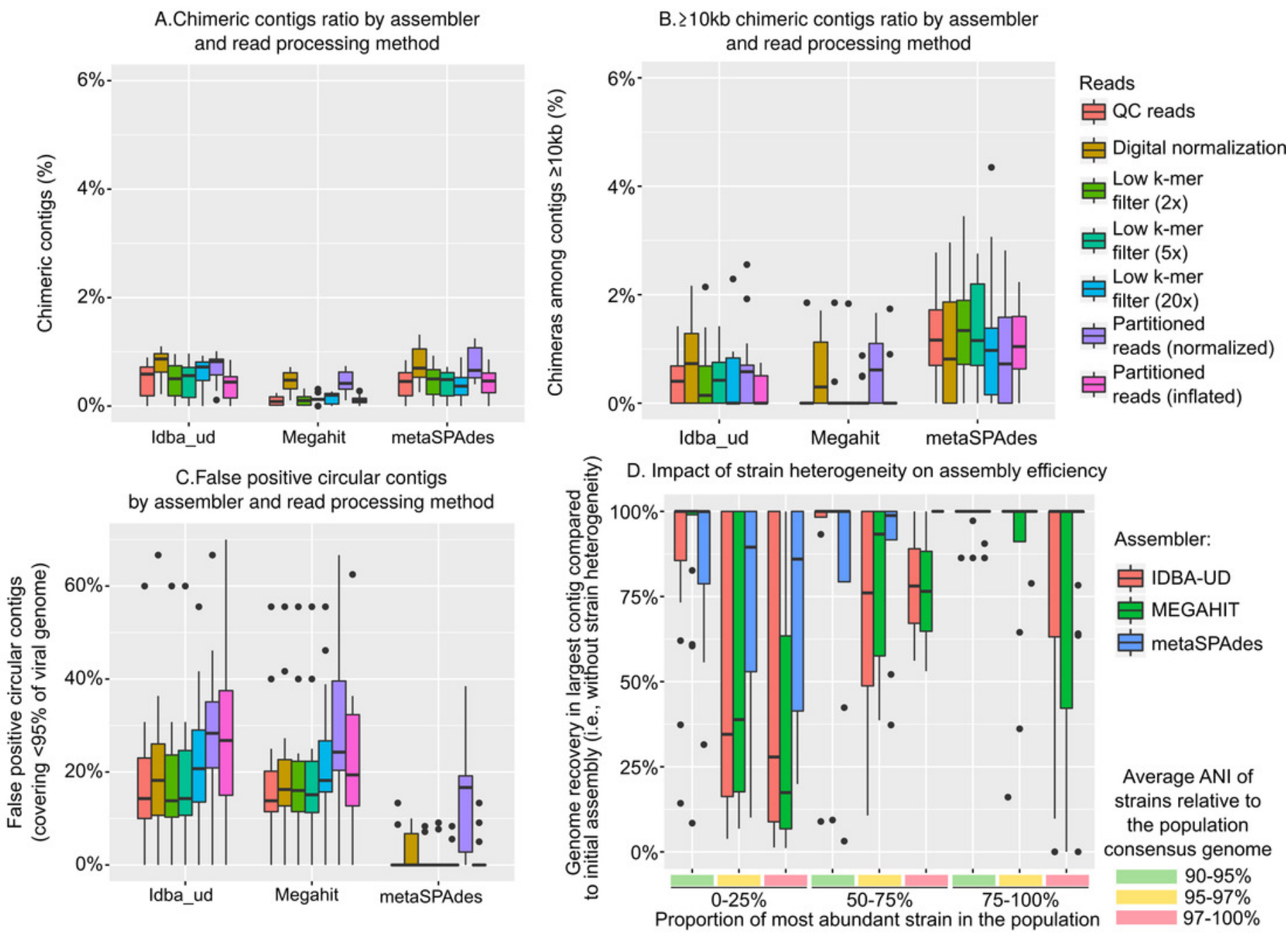


\section{Figure 3}

Figure 3. Impact of read mapping thresholds on accuracy of viral population detection.

Two parameters were investigated when parsing the mapping of individual virome reads to the population contigs pool: (i) the percentage of a contig covered by a sample to considered the contig as detected ( $x$-axis), and (ii) the percentage of identity of reads mapping to the contig (color scale). Two pools of population contigs were tested: all non-redundant contigs of $\geq 500 \mathrm{bp}$ (panels $\mathrm{A}, \mathrm{B}$, and $\mathrm{C}$ ), and all non-redundant contigs $\geq 10 \mathrm{~kb}$ (panels $\mathrm{D}, \mathrm{E}$, and F). Three metrics were calculated to evaluate the impact of mapping reads thresholds. The detection sensitivity is estimated as the percentage of "expected" genomes (i.e., genomes covered $\geq 1 \mathrm{x}$ in the sample) that were detected through mapping to population contigs (panels $\mathrm{A}$ and $\mathrm{D}$ ). The false-discovery rate corresponds to the percentage of contigs detected in a sample through mapping to population contigs, but were not associated with any genomes from the initial sample (i.e., these genomes did not provide any reads to the simulated virome, so these contigs should not be detected, panels B and E). Finally the average number of distinct population contigs detected is calculated for each individual genome initially covered $\geq 1 x$, and correspond to the number of times a single genome is "counted" (i.e. multiple contigs suggest multiple populations, even though it is really just one population, panels $\mathrm{C}$ and $\mathrm{F}$ ). 

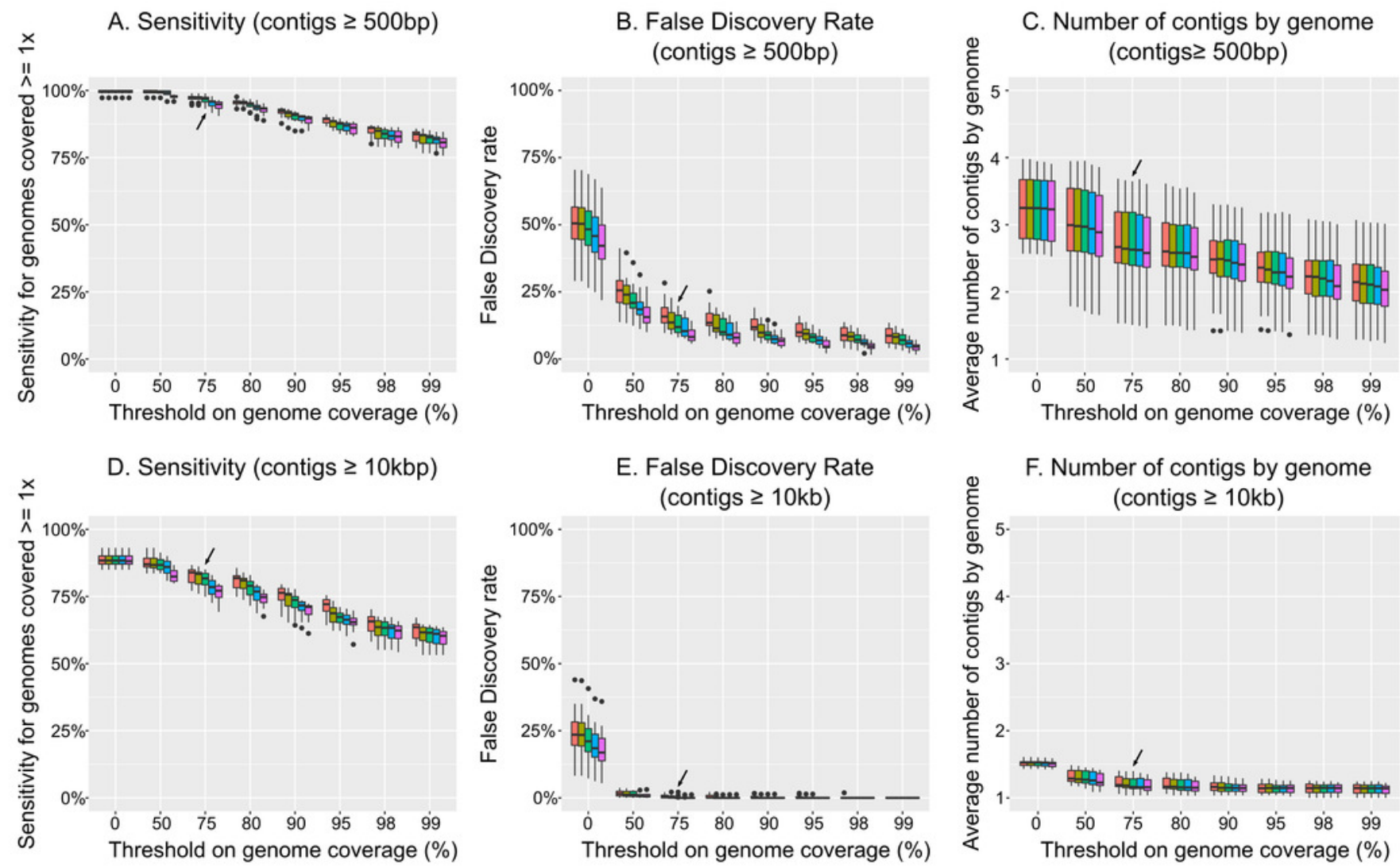

Threshold on read mapping identity percentage 追0 追80官90追95 穴98 


\section{Figure 4}

Figure 4. Estimation of alpha and beta diversity from virome-derived viral populations.

To evaluate the impact of varying sequencing depth, six viromes (highlighted in bold in panels A, B, and C), were sub-sampled at $10 \%$ (long dash) or $1 \%$ (short dash) of the original read number ("Initial" corresponds to the assemblies presented in Figure 1, 2, and 3, for which all viromes had the same initial number of reads). A. Number of genomes observed from the read mapping to viral populations. The actual number of genomes in the initial simulated community is indicated with black dots, while estimated based on viromes are colored in red. B. Comparison of Shannon diversity index from the true community composition (black dots) and estimated from the viromes (colored dots). The different estimations are based on 3 different normalization methods: counts divided by the total number of reads sequenced in the virome and the contig size ("Normalized"), counts after rarefying all viromes to the smallest dataset and normalized by contig size ("Rarefied"), and counts normalized via DESeq ("DESeq"). C. Comparison of Simpson diversity index from the true community composition and estimated from the viromes (color codes are the same as in panel B). D. Distribution of differences in Bray-Curtis dissimilarities between samples calculated from true community composition and the same dissimilarities estimated from the viromes analysis. The different normalization methods ( $\mathrm{x}$-axis) are as follows: counts divided by genome size ("Counts"), counts rarefied to the smallest dataset and normalized by contig size ("Rarefied"), counts divided by the total number of reads sequenced in the library and the contig size ("Normalized"), counts normalized by metagenomeSeq ("MGSeq"), EdgeR ("RPKM"), and DESeq ("DESeq"). E. Distribution of differences in Bray-Curtis dissimilarities between samples calculated from true community composition and the same dissimilarities estimated from virome analysis, including 6 samples sequenced at 10\%. Methods are similar as in panel D. F. Distribution of differences in Bray-Curtis dissimilarities between samples calculated from true community composition and from virome analysis, including 6 samples 
sequenced at $1 \%$. Methods are similar as in panel $D$.

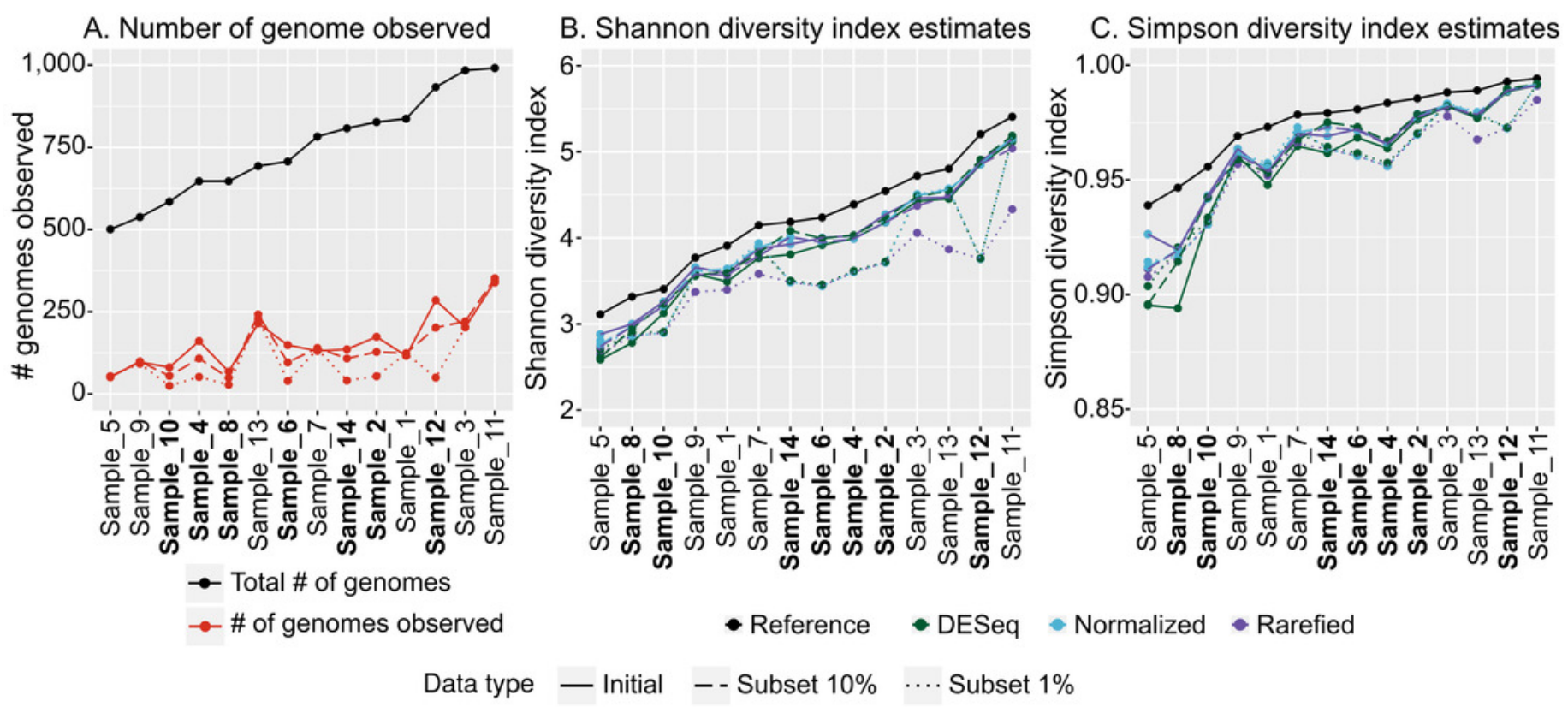

D. Bray-Curtis dissimilarities Initial sampling

E. Bray-Curtis dissimilarities Subset 10\% (subset_10)

F. Bray-Curtis dissimilarities Subset 1\% (subset_1)
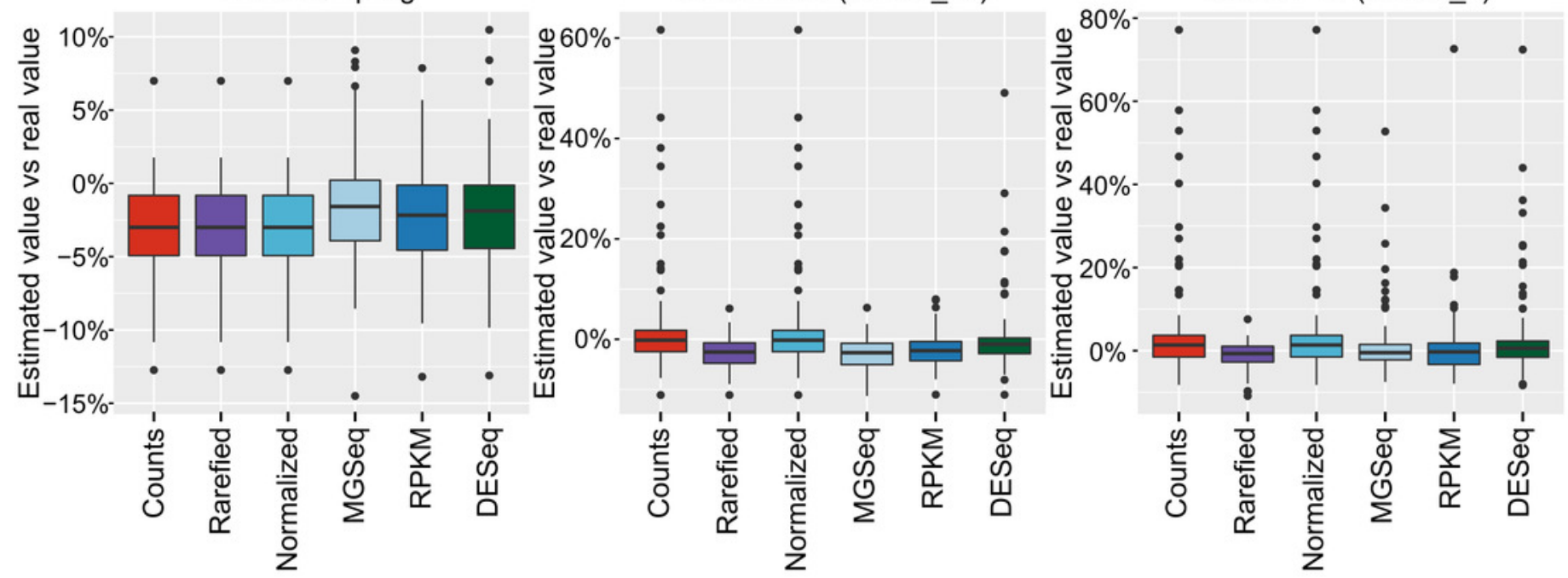